\title{
Study on Safety Demand Law of Coal Miners Based on Maslow's Demand Level Theory
}

\author{
Yao Youli, Li Xiaoxia, Peng Yingjian, Xu,Lili \\ Caol Engineering School of Shanxi Datong University
}

\begin{abstract}
In order to research on the law of man demand for safety and get safety man to know the law to validly make decision, studying on safety demand law becomes more important. According to Demand Level Theory put forward by American psychologist Maslow, applying Fuzzy mathematics, humanization demand law for safety has been researched on deeply. During every development stage, not only one demand exists, but all demand exits; just only one takes leading site. While live demand leading most important site, safety demand and others are less. Only if living demand meets satisfaction, safety demand can take up the site. So, same occupational and health standard for developed country and developing country, that is irrational. Research achievement can get safety decision maker to gather human safety demand law and truly hold human demand level in different historical and region conditions; it can help macrocosmic policy-making and microcosmic regulation to be done rightly in safety management method and safety investment; it can help decision project, technology and economy condition and human demand level to harmony. It is important for safety subject development in theory and application.
\end{abstract}

\section{Introduction}

Safety demand of mankind continually advances. Owing to difference of technology, economy level and culture structure, people in different district knowing safety is also different, but humanization demand for safety is identical[1,2]. Research on law of safety humanization demand is important for scientific policy-making and protecting safety and health of labor. What is law of humanization demand for safety? Currently, safety has not been systematically studied from the view of humanization. Based on Maslow's demand level theory, safety humanization demand is researched in the paper by fuzzy mathematics.

\section{Membership function of human demand}

Demand Hierarchy Theory put forward by American Maslow[3-5]. He pointed that human demand includes five levels, namely, from low level to high level especially living, safety, sociality, respect and selfrealization.

By Fuzzy mathematics, membership function for demand hierarchy theory is established. Method of establishing membership function includes $\mathrm{F}$ statistical method, Trichotomy, F distribution and so on[6], such as specialist marking, sorting by logic reasoning or binary contrast. In the paper F distribution method is adopted. F distribution method mainly includes rectangular distribution, half rectangular distribution, Half trapezoid distribution and trapezoidal distribution, parabolic type distribution, normal distribution and mountain distribution. Maslow's demand hierarchy theory points if low level demand is obtained next high level demand will be developed. For an individual, five demands are impossibly meet fully. Some demands may exits at the same time because human behavior is dominated by lots of demands. But, at one period, only one demand is dominating. So Maslow's level demand degree conforms to Cauchy distribution The lowest level of survival demand membership function corresponds to the small Cauchy distribution. The highest level of self-reality is corresponding to the large size of Cauchy distribution. Middle three demands, safety demand, sociality demand and respect demand meet for medium type of Cauchy distribution. Based above analysis, we can establish membership functions of five demand degree as follow:

Assuming domain $\mathrm{U}=[0,5], \quad \mathrm{U}$ expresses psychological demand level; fuzzy sets A,B,C,D,E especially express living demand degree, safety demand degree, sociality demand degree, respect demand degree self-realization demand degree; membership functions of them especially as follow:

$$
\begin{aligned}
A(x) & =\left\{\begin{array}{l}
1, x \leq 0.5 \\
\frac{1}{1+2(x-1)^{2}}, x>0.5
\end{array}\right. \\
B(x) & =\frac{1}{1+2(x-1.5)^{2}}
\end{aligned}
$$




$$
\begin{aligned}
& C(x)=\frac{1}{1+2(x-2.5)^{2}} \\
& D(x)=\frac{1}{1+2(x-3.5)^{2}} \\
& E(x)=\left\{\begin{array}{l}
\frac{1}{1+2(x-5)^{2}}, x \leq 4.5 \\
1, x>4.5
\end{array}\right.
\end{aligned}
$$

Based on above functions five demands development can be showed in Fig.1.

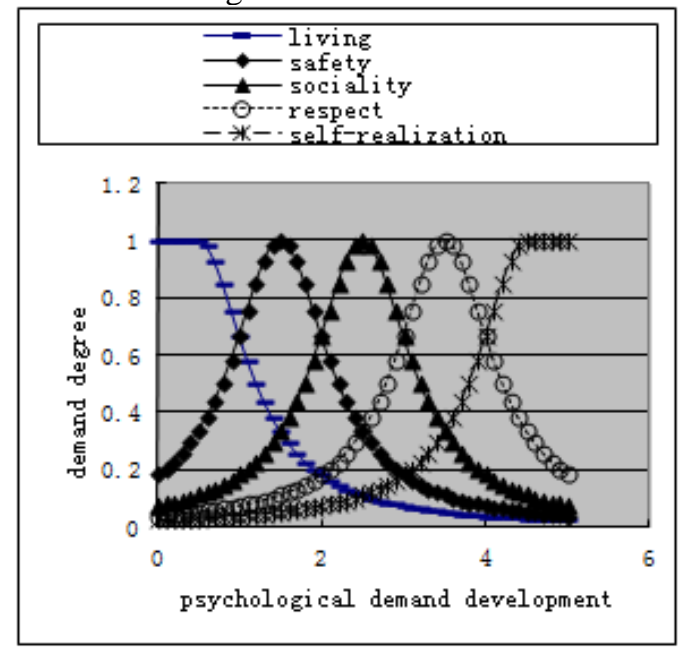

Fig.1 Psychological demand development and change

If a demand degree is larger, human will try his best to meet. So their objective satisfaction degree functions especially show as follow:

$M_{A}(x)=\frac{\int_{0}^{x} A(u) d u}{\int_{0}^{5} A(u) d u}=\frac{0.5+\frac{1}{\sqrt{2}}[\operatorname{arctg} \sqrt{2}(x-0.5)+\operatorname{arctg} \sqrt{2} \times 0.5]}{1.0005}$

$M_{B}(x)=\frac{\int_{0}^{x} B(u) d u}{\int_{0}^{5} B(u) d u}=\frac{\frac{1}{\sqrt{2}}[\operatorname{arctg} \sqrt{2}(x-1.5)+\operatorname{arctg} \sqrt{2} \times 1.5]}{0.9697}$

$M_{C}(x)=\frac{\int_{0}^{x} C(u) d u}{\int_{0}^{5} C(u) d u}=\frac{\frac{1}{\sqrt{2}}[\operatorname{arctg} \sqrt{2}(x-2.5)+\operatorname{arctg} \sqrt{2} \times 2.5]}{0.9158}$

$M_{D}(x)=\frac{\int_{0}^{x} D(u) d u}{\int_{0}^{5} D(u) d u}=\frac{\frac{1}{\sqrt{2}}[\operatorname{arctg} \sqrt{2}(x-3.5)+\operatorname{arctg} \sqrt{2} \times 3.5]}{0.799}$
$M_{E}(x)=\frac{\int_{0}^{x} E(u) d u}{\int_{0}^{5} E(u) d u}=\frac{0.5+\frac{1}{\sqrt{2}}[\operatorname{arctg} \sqrt{2}(x-4.5)+\operatorname{arctg} \sqrt{2} \times 4.5]}{0.435}$

By above functions, satisfaction degree development shows in Fig.2.

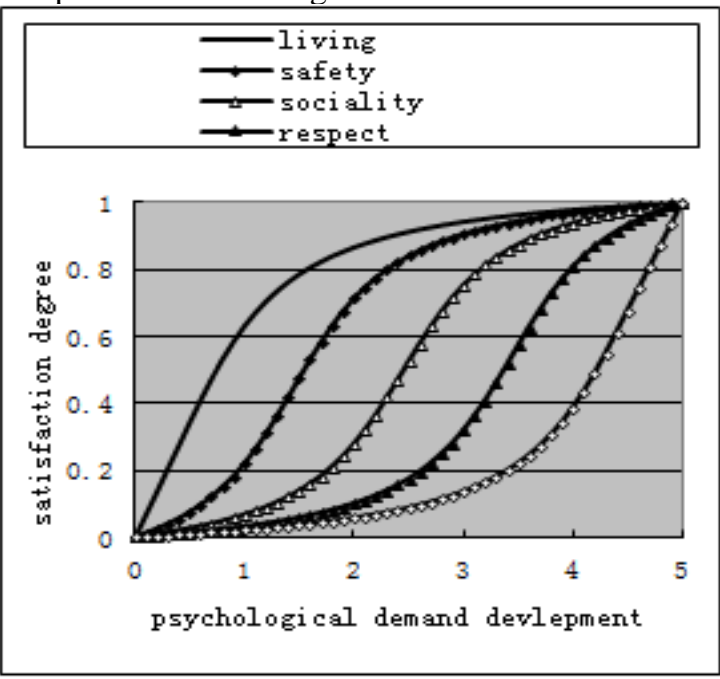

Fig.2 Objective Satisfaction Development and Change

\section{Analysis of safety demand law}

Maslow[3,7,8] thought that human demand forms a ladder from low degree to high degree. If all demands are unsatisfied lowest demand will take up ascendancy. If one demand basically is obtained its next high demand will take up ascendancy. But its specific law isn't known; it is only a notion. It also doesn't guide safety production. Only we know its law, it can be applied for human. Wherefore, we should know that demand is gradually advancing, but also we should know how to develop. In each phase of psychological demand, all demands are exist, but only one takes precedence. It can be shown in Fig.1. In $(0,1)$ phase living demand degree is highest and living demand takes precedence; in $(1,2)$ phase safety demand degree is highest; in $(2,3)$ phase sociality demand degree is highest; in $(3,4)$ phase respect demand takes precedence; in $(4,5)$ phase self-realization demand takes precedence. In $(0,0.5)$ phase living demand degree is 1 ; namely living demand takes precedence; in this phase safety demand degree is lesser and effect of safety education and training is quite bad. Only if living demand obtains safety demand degree can take precedence. Namely when psychology demand comes to $(1,2)$ of $U$, safety consciousness will actively advance. Fig. 3 is curve of cost function and safety benefit function [9-11] and at S0 point safety benefit reaches to best. Owing to $S$ increasing to $S 0$ from $S 0-\triangle S$, cost increment is lesser than safety benefit, increasing $S$ is worthy; while $S$ increasing to $S 0+\triangle S$ from $S 0$, cost increment is larger than safety benefit, and in the view of economics increasing S isn't worthy; but in the view of human safety demand safety input would continue to be done, though it isn't worthy in the view of economics; with economy developing and safety input will gradually 
increase; current some developed countries is so in such way.

In Maslow's demand hierarchy theory, safety demand is in second level. Namely, only if living demand obtains, human will consciously demand safety. When living demand takes precedence, human only revolts in conditioned flex way for other injury, but consciously retort; human passively resists only for living, but not protect by safety technology. Such instinct action is impossible to void injury and death; under such conditions injury and death is regarded as acceptable risk; so safety demand degree is lower (shown in Fig.1). Some years ago, one coal mine in Guaizhou province, its safety situation is quite bad and accidents repeatedly happened; while safety lawmen and specialists decided to shut this coal mine, harrowing situation occurred, which local tearing people solicited not to shut their coal mine and they said they demand bread by this coal mine. This example well showed while living demand taking precedence safety demand and other demand also exist but not be important. So, same occupational safety standard for developing countries and developed countries is evidently irrational. Research result will strike back some international argument.

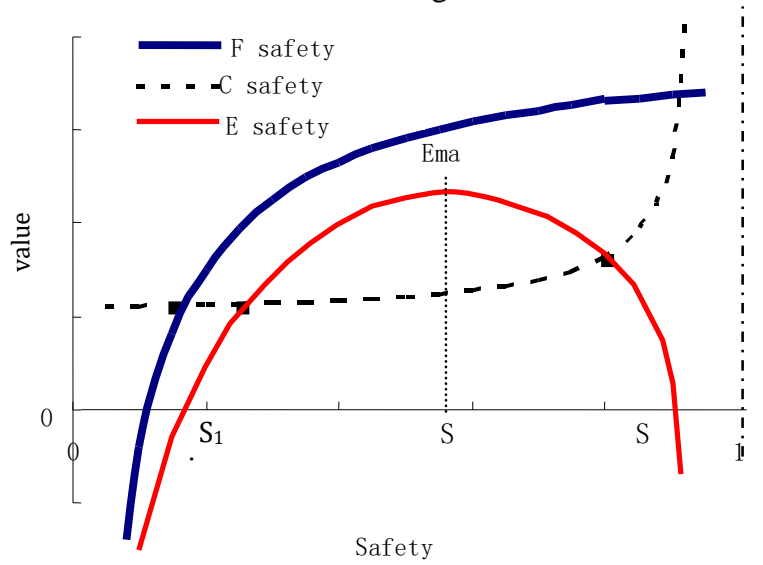

Fig.3 Safety cost function and efficiency function.

\section{Conclusion}

Research result can get safety decider to know human safety demand law and to make policy in macrocosm and to control in microcosm. Its function as follow:

(1) It is helpful for safety management.

Currently, emphases of safety science research depend on new technology, new material and new process to advance safety level. However, safety management is more important to advance safety level. It is insufficient to only depend ideological education to advance human safety consciousness and technological training to advance safety skill. So, knowledge of human safety demand law is helpful for safety management goal.

(2) It is helpful for macrocosm policy and microcosm control.

By local people safety demand situation, safety macrocosm policy more accord with practice. Policy by experience can result in two kickbacks. One is safety object made to be too high because of immature technology and inadequate economy; it can lead to investment to be too large; Object is too high to reach in the short run; it can bring negative psychological influence; it can lead to safety benefit to be decreased. Two is safety object made to be too low; workers have to accept unacceptable risk; it can cause their dissatisfaction and bring passive revolt psychology. Insufficiency safety input can result in lower safety level. It is irresponsible for workers.

\section{References}

1. Tian Shuicheng, Li Hongxia, Feng Changgen. Analysis on Safety Demand and Supply[J]. Labor Protection Technology. 2006.6:9-11.

2. Xu Kaijing. Safety Demand is Main Cause to Define Citizen Consumption[J]. Qinhai Social Science. 2001.1:34-37.

3. $\mathrm{Xu}$ Jiang, $\mathrm{Wu}$ Qiong. Safety Management[M]. Beijing: Aviation Industry Press. 1993.

4. Jia Xiaoming, Zhao Shuming. Reflective Thinking of Maslow Demand Theory[J]. Modern Management Science. 2004.6:12-16.

5. Ren Huai. Philosophy thought of Confucianism and Maslow's Safety Demand[J]. Liaoning Engineering and Techonolgy University Journal. 2007.5:239-241.

6. Yang Lunbiao, Gao Yingyi. Fuzzy Mathematics Theory and Application[M]. Guangzhou: Huanang Polytechnic Institute Press. 2002.1.

7. Chen Senyao. Safety Management Theory[M]. Beijing: Aviation Industry Press. 1996.

8. Chen Baozhi. Safety Theory[M]. Beijing: Metallurgical Industry Press. 1995.

9. Luo Yun. Safety Economics[M]. Beijing: Economy Science Press. 2004.

10. Song Dacheng. Enterprise Safety Economics (loss part)[M]. Beijing: Weather Press. 2000.

11. Albert Sese, Alsonso L.Palmer. Occupational Safety and Health in Spain. Journal of Safety Research. 2012.33:511-525. 